\title{
Atuação do enfermeiro em laboratório de análises clínicas
}

\author{
Nurse's performance in a clinical analysis laboratory \\ Desempeño de la enfermera en un laboratorio de análisis clínico
}

\begin{abstract}
RESUMO
Objetivou-se descrever as atribuições dos enfermeiros que atuam em um laboratório de análise clínica da grande Florianópolis, do estado de Santa Catarina, Brasil. Trata-se de um estudo qualitativo, de campo, de caráter descritivo - exploratório. 0 desenho metodológico foi executado em 5 fases: fase 1: autorização da pesquisa; fase 2: validação do instrumento de coleta de dados por especialistas na área; fase 3: entrega do termo de consentimento livre esclarecido aos sujeitos da pesquisa, o público alvo foram os enfermeiros lotados no setor de laboratório de análises clínicas; fase 4: coleta de dados após aplicação do questionário aberto contendo 19 questões; e fase 5: recolhimento dos questionários e interpretação e análise dos dados. 0 estudo demonstrou que o enfermeiro possui atribuições nas três fases distintas do processo, desenvolvendo atribuições privativas do Enfermeiro, tanto na parte assistência quanto na parte gerencial. Além disso, evidenciou-se a importância de utilizar instrumentos que sustentem suas práticas para a tomada de decisão e a importância da busca de conhecimento constante nessa área que cada vez mais o profissional Enfermeiro vem sendo inserido.
\end{abstract}

DESCRITORES: Cuidados de Enfermagem; Laboratórios; Fase Pré-Analítica; Enfermeiro.

\section{ABSTRACT}

The aim was to describe the duties of nurses working in a clinical analysis laboratory in Florianópolis, in the state of Santa Catarina, Brazil. It is a qualitative, field, descriptive - exploratory study. The methodological design was carried out in 5 phases: phase 1: research authorization; phase 2: validation of the data collection instrument by specialists in the field; phase 3: delivery of the informed consent form to the research subjects, the target audience was nurses working in the clinical analysis laboratory sector; phase 4: data collection after application of the open questionnaire containing 19 questions; and phase 5: collecting the questionnaires and interpreting and analyzing the data. The study demonstrated that the nurse has assignments in the three distinct phases of the process, developing private assignments of the Nurse, both in the assistance part and in the managerial part. In addition, the importance of using instruments that support their practices for decision making was evidenced and the importance of the search for constant knowledge in this area that more and more the professional Nurse has been inserted.

DESCRIPTORS: Nursing Care; Laboratories; Pre-Analytical Phase; Nurse.

\section{RESUMEN}

El objetivo fue describir las funciones de las enfermeras que trabajan en un laboratorio de análisis clínicos en Florianópolis, en el estado de Santa Catarina, Brasil. Es un estudio cualitativo, de campo, descriptivo-exploratorio. El diseño metodológico se llevó a cabo en 5 fases: fase 1: autorización de la investigación; fase 2: validación del instrumento de recolección de datos por especialistas en la materia; fase 3: entrega del formulario de consentimiento informado a los sujetos de investigación, el público objetivo fueron enfermeras que trabajan en el sector de laboratorios de análisis clínicos; fase 4: recopilación de datos después de la aplicación del cuestionario abierto que contiene 19 preguntas; y fase 5: recopilación de los cuestionarios e interpretación y análisis de los datos. El estudio demostró que la enfermera tiene asignaciones en las tres distintas fases del proceso, desarrollando asignaciones privadas de la Enfermera, tanto en la parte asistencial como en la gerencial. Además, se evidenció la importancia de utilizar instrumentos que apoyen sus prácticas para la toma de decisiones y la importancia de la búsqueda del conocimiento constante en esta área que cada vez más se está insertando la Enfermera profesional.

DESCRIPTORES: Cuidado de Enfermería; Laboratorios; Fase Preanalítica; Enfermera.

RECEBIDO EM: 14/06/2020 APROVADO EM: 16/06/2020 


\section{Josué Prigoli}

Enfermeiro. Secretaria Municipal de Saúde da Prefeitura de Florianópolis.

ORCID: 0000-0003-0365-8863

\section{Vanessa Costa}

Enfermeira Laboratório de Análises Clínicas Santa Luzia.

ORCID: 0000-0002-9138-4227

\section{Gisele Francisca de Souza}

Enfermeira. Especialista em Emergência e Urgência. MBA em Gestão de Pessoas e Liderança de Equipes. Dasa - Laboratório Médico Santa Luzia.

ORCID: 0000-0002-7981-0983

\section{Catia Cristina Santiago da Silva Praxedes Branco}

Bibliotecária. Especialista em Gestão Estratégica de RH. Mestre em Ciência da Informação. Dasa- Laboratório Médico Santa Luzia.

ORCID: 0000-0001-5702-1462

\section{Mayara Marta Rodrigues}

Enfermeira na Prefeitura Municipal de São José. Doutoranda do Programa de Pós-Graduação em Enfermagem UFSC.

ORCID: 0000-0003-1353-125X

\section{Thayse Rosa}

Enfermeira. Mestre em Enfermagem. Doutoranda da Universidade do Extremo Sul Catarinense - UNESC, Criciúma, SC. Professora Titular no Centro Universitário para o Desenvolvimento do Alto Vale do Itajaí - UNIDAVI, Rio do Sul, SC. Professora Titular na Faculdade de Santa Catarina - FASC, São José, SC.

ORCID: 0000-0002-4623-9362

\section{INTRODUÇÃO}

0 laboratório tem por objetivo fornecer dados diagnósticos, que auxiliam na conduta terapêutica do paciente. Os dados são obtidos da análise da matéria orgânica de um determinado paciente, por exemplo: urina; fezes; saliva; sangue arterial e venoso; amostras de tecido e líquor. A coleta da matéria orgânica corresponde ao processo de maior complexidade por possuir diferentes técnicas, umas menos invasivas, geralmente com baixo risco e outras mais invasivas que podem apresentar um risco significativo. Nesse processo, o enfermeiro é capacitado e possui competência para fornecer os cuidados diretos ao paciente, supervisionar e capacitar os profissionais que realizaram as coletas e atuar como gestor no processo administrativo nos locais onde forneçam assistência à saúde ${ }^{(1)}$.

Os laboratórios de análises clínicas utilizam o processo dinâmico que é fundamentado em três fases distintas: a fase pré-analítica, analítica e pós-analítica. A primeira, ou seja, a fase pré-analítica corresponde à preparação do cliente, a técnica da coleta, aos produtos e materiais utilizados, ao armazenamento e ao transporte do material ${ }^{(1)}$.

Nesse contexto, o enfermeiro desenvolve diversas atividades nos Laboratórios de Análises clínicas, por exemplo: o gerenciamento da assistência de enfermagem; organização da instituição; protocolos; procedimentos operacionais padrão (POPs); elaboração de questionários para a coleta de dados; orientações ao cliente; manuais; administração do setor; treinamentos dos profissionais de enfermagem, orientações e intervençôes no preparo dos pacientes antes, durante e depois da coleta. Estas atribuições demandam de visão ampla e científica pelo profissional enfermeiro, englobando não somente os aspectos técnicos, éticos e legais dos serviços prestados, mas também o conhecimento acerca dos trabalhos da enfermagem neste campo ${ }^{(2)}$.

A Portaria CVS-13, de 04-11-2005, aprova Norma Técnica que trata das condições de funcionamento dos Laborató- rios de Análises e Pesquisas Clínicas, Patologia Clínicas e Congêneres, descreve em seus parágrafos a definição dos procedimentos de coletas de material humano e define os profissionais que podem atuar na área de coleta e na sua coordenação. No seu Art. 4.441 dos recursos humanos, autoriza atuação dos profissionais de nível superior para atuar no setor, são eles: médicos e enfermeiros; farmacêuticos; biomédicos; biólogos; e químicos ${ }^{(3)}$.

Mesmo com fomentação legal para exercício da profissão, pode-se observar que na graduação de enfermagem, o conhecimento que o enfermeiro adquire está voltado para outros setores da saúde, porém, de forma geral abrange a necessidade encontrada nas diversas funções que um laboratório de análises clínicas necessita. Na graduação de enfermagem não há matéria específica na grade direcionada à atuação dentro de um laboratório de análises clínicas. $\mathrm{Na}$ parte prática, nos estágios curriculares, também não é contemplada essa área ${ }^{(2)}$.

Dentro de um laboratório de análises clínicas, na fase pré-analítica muitos 
procedimentos de coletas são privativos do enfermeiro, conforme o Decreto n. ${ }^{\circ}$ 94.406/1987, Art. " 8 o , O enfermeiro que domina a sua técnica e o conhecimento que envolve a assistência de enfermagem a este tipo de cliente, é aquele capaz de dar melhor qualidade no serviço prestado".

Esse estudo se torna viável devido a sua relevância acadêmica e social, podendo fornecer maior conhecimento nessa área, auxiliando os profissionais e acadêmicos de enfermagem olharem para esse setor que se encontra em crescimento e saber que é uma possibilidade de atuação, e principalmente de evidenciar sobre as principais atribuições que os enfermeiros realizam atualmente neste setor, uma vez que, os profissionais devem realizar seus serviços dentro dos mais altos padrões de qualidade e dos princípios da ética/bioética, tendo em conta que a responsabilidade da atenção à saúde não se encerra com o ato técnico, mas sim, com a resolução do problema de saúde, tanto em nível individual como coletivo. Já do ponto de vista coletivo, contribuirá para melhoria da assistência de enfermagem atingindo diretamente ao público assistido por eles. Sendo assim, este estudo possibilita conhecimento aos profissionais, norteando os cuidados de enfermagem.

A pesquisa foi realizada com a participação dos profissionais de enfermagem de uma rede de laboratórios de análises clínicas, na iniciativa privada atuante na grande Florianópolis, Capital de Santa Catarina, Brasil. Que tem como pergunta de pesquisa: "Quais são as atribuiçõoes do enfermeiro prestadas aos clientes em um laboratório de análises clínicas da grande Florianópolis, no Estado de Santa Catarina, Brasil? Dado o exposto, objetivou-se conhecer as atribuições exercidas pelos enfermeiros que atuam em uma rede de laboratório de análise clínica da grande Florianópolis, Santa Catarina - Brasil.

\section{METODOLOGIA}

Trata-se de um estudo qualitativo de caráter descritivo e exploratório que busca descrever o papel do profissional enfermeiro que atua em laboratórios de análises clínicas.

A pesquisa ocorreu em um laboratório de análises clínicas localizado na grande Florianópolis, no estado de Santa Catarina. Trata-se de uma instituição privada organizada por 1 matriz e 16 filiais.

Foram convidados para esta pesquisa a totalidade de 13 enfermeiros, lotados em unidades laboratoriais, com base nos critérios de inclusão, os quais foram estabelecidos em: profissionais que tenham mais de 06 meses de experiência e que atuassem diuturnamente no período da referida pesquisa. Já os critérios de exclusão foram: enfermeiros que não fosse possível o contato após 3 tentativas; que não tivessem interesse em participar da pesquisa e profissionais que estivessem de férias, licença ou de atestado durante o período de coleta de dados, fatores que resultaram nas 08 participantes informadas.

A entrada no campo ocorreu por meio da apresentação do projeto finalizado à gerência de enfermagem da instituição, informando os aspectos relacionados à pesquisa. $\mathrm{O}$ desenho metodológico apresenta 5 fases, sendo 1 fase pré-coleta de dados conforme descrito abaixo:

Fase 1 - Autorização para desenvolvimento da pesquisa por meio de ofício apresentado à gerente de enfermagem da instituição na qual a pesquisa foi desenvolvida, com o intuito de analisar e o aprovar, e seguir para o parecer do Comitê de Ética e Pesquisa da Universidade Paulista (CEP/UNIP). Após as respectivas aprovações, deu-se início às outras etapas metodológicas subsequentes;

Fase 2 - Validação do Instrumento após envio de TCLE e carta convite para as juízas. Nesta fase, o instrumento de validação foi construído com o objetivo de avaliar 19 questões abertas do instrumento de coleta de dados. Em seguida, foram convidados 3 juízes para participarem da validação do instrumento antes de dar sequência à coleta de dados. Os critérios de inclusão para participação da pesquisa adotada foram: ter experiência consolidada no setor e/ou especialização na área (análise realizada através do currículo
Lattes). Os profissionais que se enquadrassem nas características exigidas foram contatados previamente via telefone/e-mail e, posteriormente, foram enviadas as cartas convite e o TCLE, juntamente com o instrumento para validação com caracterização dos juízes e informações de esclarecimentos sobre como realizar a avaliação. No processo de validação, cada atividade foi avaliada quanto à pertinência e à concordância. Após o recebimento dos instrumentos, os dados foram analisados. Para cada item avaliado como não pertinente por algum juiz, esse item e as razões apontadas para sua não pertinência eram apresentados aos demais juízes para que a concordância fosse julgada. Importante ressaltar que o anonimato dos juízes foi mantido em todas as fases. Três juízes que aceitaram previamente participarem do estudo validaram o instrumento. A validação ocorreu em maio de 2019;

Fase 3 -Nesta fase os pesquisadores se apresentaram para cada sujeito do estudo individualmente, convidaram a participar do estudo e esclareceram a natureza de tal, sua justificativa, seus objetivos, os possíveis benefícios e riscos da mesma, a importância da participação de cada um, a garantia do anonimato e, por fim, que os dados obtidos serão utilizados para fins científicos, realizarão a leitura, discussão e assinatura do TCLE;

Fase 4 - A coleta de dados foi realizada utilizando-se como instrumento um questionário aberto, elaborado pelos pesquisadores, contendo 19 questões. O instrumento foi preenchido pelos sujeitos de pesquisa no horário de em que estivessem disponíveis, sendo acordado que os pesquisadores iriam retornar após 1 semana para retirada do questionário respondido;

Fase 5- Esta fase consiste no recolhimento dos questionários pelos pesquisadores em cada turno do setor. Foram descartados aqueles que não entregaram o questionário respondido após 3 tentativas dos pesquisadores. Realizada a interpretação e análise qualitativa.

Após a coleta dos dados, a organização do banco de dados foi feita por meio de uma planilha no programa Microsoft Ex- 
$\mathrm{cel}^{\circ}$. Posteriormente, foram realizadas análises descritivas dos dados através da modalidade temática ${ }^{(5)}$ e os resultados foram organizados em categorias. Todavia, juntamente com a literatura e em consonância com a teoria de Virginia Henderson ${ }^{(6)}$, as respostas foram correlacionadas.

\section{RESULTADOS E DISCUSSÃO}

A população que compõe a presente pesquisa é de 06 enfermeiros, foram retiradas da pesquisa com base nos critérios de exclusão, 01 se desligou da empresa, 01 que estava no período de férias, 02 tinham apenas 3 meses de vivência no setor e 03 não entregaram os questionários nas várias tentativas realizadas.

$O$ perfil dos profissionais de enfermagem da rede de laboratórios em que ocorreu a presente pesquisa segue descrito conforme podemos observar: todas as pesquisadas eram do sexo feminino e trabalhavam no período diurno; quanto à idade, faixa etária dos 25 aos 37 anos; quanto ao tempo de atuação na área, há profissionais entre 06 meses e 10 anos de vivência; nenhum dos sujeitos de pesquisa possuíam vivência em laboratório considerando outras instituições; quanto aos títulos, apenas 02 são especialistas, porém em outras áreas de atuação.

O Enfermeiro tem seu espaço garantido na equipe multiprofissional, uma vez que possui alguns procedimentos privativos à sua formação. A determinação da Lei do Exercício Profissional de Enfermagem estabelece procedimentos privativos do enfermeiro, e na presença de técnicos e auxiliares de enfermagem determina a necessidade de supervisão do enfermeiro. Entretanto, apesar da área laboratorial ser um setor que a enfermagem vem ganhando espaço nas últimas décadas, observamos poucos estudos de enfermagem sobre o assunto, o setor está em fase de adequação com as normas do Conselho Federal de Enfermagem (COFEN), trazendo novas oportunidades de campo de atuação para a enfermagem, mesmo assim, é comum ter o profissional técnico de enfermagem no setor, sendo supervisionado por profissionais de outras áreas ${ }^{(7)}$.
A próxima etapa da análise e interpretação dos dados ocorreu após organização e leitura exaustiva dos resultados para melhor compreensão, serão apresentados nos próximos subtítulos 1 categoria e 4 subcategorias, tais quais: Principais Atribuições do Enfermeiro no laboratório de análises clínicas (Fase pré-analítica, Analítica, Pós-analítica; Atividades gerenciais).

\section{Principais atribuições dos profissio- nais de enfermagem no laboratório de análises clínicas}

A área laboratorial de análise clínica, requer do profissional enfermeiro a forma diferenciada do padrão tradicional de modelo Biomédico, a assistência está organizada por rotinas e protocolos para o guiarem, além disso, a modernização tecnológica e novas técnicas surgem constantemente, exigindo desse profissional conhecimento técnico e científico atualizado. $\mathrm{O}$ atendimento prestado neste setor vem sendo adequado às políticas públicas de cuidados individualizado e humanizado, focados não apenas nos problemas, mas no indivíduo como um todo ${ }^{(2)}$.

No que se refere especificamente à conduta do profissional enfermeiro no âmbito do laboratório de análises clínicas, A sua atuação está amparada na Lei n. ${ }^{\circ} 7.498 / 86$ do exercício da profissão, no Decreto n. ${ }^{\circ}$ 94.406/87 que regulamenta suas atribuições e normas específicas do setor, como CVS-13/2005 que trata das condições de funcionamento dos laboratórios de análises clínicas e habilita os profissionais enfermeiros técnicos e auxiliares de enfermagem entre outros profissionais, para atuar nesta área ${ }^{(4)}$.

Mesmo assim, ainda é comum ter locais que não se adequem quanto à presença do Enfermeiro, outro fator existente é de profissionais da enfermagem que desconhecem este local como uma área de atuação para enfermeiro. Desta forma, fica claro a obrigatoriedade do profissional neste setor e conhecer as atribuições nessa área são necessárias para a classe em questão. As atribuições do Enfermeiro dentro do laboratório de análises clínicas são voltadas para as fases: pré-analítica, analítica, pós-analítica e atividades gerenciais.
- Fase pré-analítica: as atribuições realizadas pelo Enfermeiro na fase pré-analítica segundo os sujeitos da pesquisa incluem:

"O enfermeiro pode atuar, desde o registro adequado dos protocolos de exames, coleta dos mesmos, e ainda do Setor da Centrifugação, no qual são acondicionadas elou centrifugadas as amostras até o transporte das mesmas." (Enfermeira 1)

"O enfermeiro participa desde a recepção dando orientação para $o$ cliente até a realização da coleta, atentar para sinais de TEP e Grades na cama." (Enfermeiro 2)

"Orientação e coleta de materiais. Organização de treinamentos para equipes." (Enfermeira 4)

"O enfermeiro participa de todo o pré- analítico, nas instruçóes de coletas de exames, no cadastro do material e em todo preparo para coleta." (Enfermeira 5)

Podemos observar que a maioria das enfermeiras relatou a atuação do enfermeiro em todo o processo da fase pré-analítica e demonstram estar bem embasadas quanto suas atribuiçõos. As pesquisadas citaram tanto atribuições assistenciais quanto gerenciais.

Sendo assim, o enfermeiro tem competência e habilitação legal para desenvolver esses procedimentos, outra atividade que pode ser desenvolvida por ele é a centrifugação.

O setor de centrifugação é o local onde separamos as amostras de exames coletados por setor de análise como: hematologia, bioquímica, urinálise, parasitologia, imunoquímica. Ali todos os materiais são manipulados corretamente, acondicionados e separados com segurança. Nessa fase pré-analitica se garante a confiabilidade, segurança e pontualidade das amostras coletadas. $\mathrm{O}$ enfermeiro participa diretamente com os treinamentos da equipe relacionados à manipulação correta do material, tempe- 
raturas das amostras, acondicionamento dos mesmos, visando garantir minimizar os riscos de contaminação, até seu transporte correto, mantendo a temperatura exata para cada setor de amostras nas suas devidas maletas. A maioria das amostras segue uma rotina de transporte de amostra biológico entre os laboratórios ${ }^{(8)}$.

Considerando ainda as atribuições da fase pré-analítica, além da assistência direta, o Enfermeiro também tem atribuições gerenciais como verifica-se abaixo:

"Nem sempre de forma direta. Mas tem sua participação em acréscimo de informações, elaboração de alguns documentos e aplicação de novos processos." (Enfermeira 6)

"O enfermeiro participa desde o planejamento e supervisão da assistência até as avaliaçôes e cuidados tanto do paciente como dos serviços." (Enfermeira 3)

Fica evidente que a supervisão de enfermagem é uma atribuição muito importante para melhoria da qualidade da assistên- cia prestada aos clientes e envolve toda fase pré-analítica. Além da supervisão como atribuição de extrema importância na parte gerencial temos também atribuições citadas, e não menos importantes, como: orientações, educação continuada, treinamento e capacitações.

Sendo assim, quanto mais o Enfermeiro conseguir capacitar sua equipe, seja por meio de educação continuada, elaboração de POPs ou estabelecendo novos processos baseados em evidências científicas, mais estará contribuindo para a segurança do paciente e dos profissionais que ali trabalham. Conforme estudo ${ }^{(9)}$, os perigos existentes nos laboratórios incluem os riscos químicos, físicos, biológicos, ergonômicos e os profissionais devem se manter atualizados para a prevenção de acidentes de trabalho.

Para melhor compreensão, foi elaborado Quadro abaixo onde estão descritas as atribuições privativas do enfermeiro, contendo suas respectivas referências legais.

Segundo relatos das sujeitas de pesquisa quanto aos procedimentos privativos que realizam em sua rotina incluem:

"Procedimentos tais como: coletas por meio de cateterismos vesicais de alívio; coletas de Colpocitologias; gasometrias arteriais; etc." (Enfermeira 1)

\section{"Sondagem vesical, gasometria ar- terial, Colpocitologia oncótica, ad- ministração de medicamentos em provas hormonais, entrega de laudo HIV e liderança." (Enfermeira 5)}

Observa-se que as sujeitas de pesquisas estão em concordância quantos aos procedimentos privativos do enfermeiro, apenas uma citou administração de medicamentos em provas hormonais, embora não encontramos na literatura amparo legal que determine que essa atribuição é privativa do enfermeiro, é possível que essa atividade seja executada na rotina normatizada por um POP do local pesquisado, afinal, administração de insulina e clonidina incluem riscos relativos à administração, podendo causar hipotensão, alterações cardiovasculares, necessitando de uma estrutura montada para situações de urgência e emergência, necessitando do enfermeiro um aparato para a correta tomada de decisão e coordenação da equipe caso

\section{Quadro 1. Atividades privativas do enfermeiro. São José, SC, Brasil, 2019}

ATIVIDADES PRIVATIVAS DO ENFERMEIRO (D EC R E TO N 94.406/87

\begin{tabular}{|c|c|c|}
\hline 01 & Processo de enfermagem & RESOLUÇÃO COFEN- 514/2016 \\
\hline 02 & Aprazamento de Prescrição Médica & PARECER COREN -SP- 036/2013 \\
\hline 03 & Classificação de risco & RESOLUÇÃO COFEN-423/2012. \\
\hline 04 & Cateterismo vesical de alívio e demora & RESOLUÇÃO COFEN-450/13 \\
\hline 05 & Punção de Port-a-Cath & PARECER COREN-DF-30/2009. COREN-DF-10/09 \\
\hline 06 & Punção de Veia na Jugular & PARECER COREN-MG-31/06 \\
\hline 07 & Passagem, cuidados e manutenção de PICC & RESOLUÇÃO COFEN-58/2001 PARECER COFEN- 15/2014 PARECER \\
\hline 08 & COFEN-243/2017 \\
\hline 09 & Coleta de Gasometrismo umbilical. & RESOLUÇÃO COFEN- 388/11 \\
\hline 10 & Retirada de Introdutor Vascular & PARECER COREN-SP-007/2012 \\
\hline 11 & Retirada de Drenos & PARECER COREN-SP-053/2013 \\
\hline 12 & Terapia de Nutrição Parenteral & RESOLUÇÃO COFEN- 453/2014 \\
\hline 13 & Sondagem/ Cateterismo Nasoenteral & RESOLUÇÃO COFEN-0453/2014 \\
\hline 14 & Consulta de enfermagem & RESOLUÇÃO COFEN nº 514/2016 \\
\hline 15 & Colpocitologia oncótica pelo método de Papanicolau & RESOLÇÃO COFEN-381/2011 \\
\hline 16 & Entrega de laudos HIV & PORTARIA MINISTÉRIO DA SAÚDE N 151/2009 \\
\hline
\end{tabular}




\section{artigo}

ocorra uma intercorrência, onde o tempo é fundamental.

Para as Enfermeiras pesquisadas, as atribuições privativas do enfermeiro mais complexas e/ou que possuem maior dificuldade em realizar são:

"Gasometria de paciente pediátrico, como se trata de uma criança, não entende que precisa ficar com membro parado, muitas vezes precisamos imobilizar a criança. (Enfermeira 5)

"Sondagem vesical de alivio, pacientes com cirurgia no períneo se tem uma certa dificuldade para localizar a uretra." (Enfermeira 3)

"Sondagem vesical em $R N$, devido ao acolhimento aos pais."

(Enfermeira 4)

"A Sondagem de criança/Bebês o qual o orifício da uretra é de difícil acesso devido o tamanho $e$ localização da uretra e vagina, por estarem próximos. Sendo que a contração da musculatura compromete também o sucesso do procedimento." (Enfermeira 6)

"Gasometria. Localização da artéria." (Enfermeira 2)

Como pode-se observar, metades referiu dificuldade em realizar procedimentos no público pediátrico, se tratando de procedimentos privativos do enfermeiro, onde por si só o procedimento envolve uma complexidade maior devido ser invasivo.

Quando atendemos crianças que ainda não entendem a necessidade de se realizar o procedimento ou não colaboram devido a diversas causas, podem colocar o procedimento em risco, sendo necessário imobilizar a criança. Isso ocorre em crianças pequenas, mas somente em casos de extrema necessidade. Já com as crianças maiores, uma boa orientação e informação do exame ou tratamento que será realizado podem tranquilizá-las e obter uma boa colaboração. A restrição de movimentos é feita com os seguintes objetivos: facilitar a realização de exames e a aplicação de tratamentos, proteger a criança contra acidentes devido a sua agitação, evitar que a criança retire com as mãos sondas, drenos, coletores e aplicações de soro, evitar que a criança provoque lesões na área do tratamento, entre outros ${ }^{(10)}$.

Avaliando os riscos relacionados ao procedimento e que podem levar danos ao paciente, observam-se os relatos abaixo:

"Nas sondagens vesicais, pode ocorrer desde alguma lesão a nivel de uretra, até perfuração da bexiga; na colpocitologia, podem ocorrer lesões elou traumas a nivel de canal vaginal, até o colo uterino; na gasometria, pode ocorrer lesóes vasculares e até mesmo necrose de tecidos; etc." (Enfermeira 1)

\section{"Gasometria - lesão de nervo. Impor- tante conhecer a anatomia, e saber sinais que indicam lesão.Sondagem vesical - Estenose de uretra. Saber utilizar material correto, calibre adequado de sonda, contaminação por microorganismos. Administração de medicamentos. Administrar dosa- gem errada." (Enfermeira 5)}

Por mais que o procedimento ocorra frequentemente nas rotinas de enfermagem, os cuidados não podem ser negligenciados pelo profissional, demandando de conhecimento científico e atualização constante, com risco de prejuízo ao quadro de saúde do paciente.

Os riscos relatados demonstram a importância dos procedimentos serem realizado por um profissional com conhecimento técnico/científico e treinamento relacionado a o procedimento, como também na colpocitologia, mesmo sendo um procedimento indolor e rápido, o exame preventivo pode, no máximo, causar um pequeno desconforto que diminui se a mulher conseguir relaxar e se o exame for realizado com boa técnica e de forma delicada.

Esses conhecimentos são necessários para correta análise da ectocérvice, para descartar lesões, possíveis ISTs, lesão por HPV, odor característico das vulvovaginites, avaliação da coloração saudável do colo, entre outros achados que o profissional enfermeiro é habilitado desde sua formação, devido a conhecimentos adquiridos na grade curricular, como: anatomia, fisiologia, semiologia e semiotécnica, estágio (prático) entre outras.

Várias situações que demandam de uma rápida tomada de decisão podem surgir no dia a dia do Enfermeiro que atua no laboratório de análises clínicas. Este é um setor que atende tanto clientes saudáveis que vão fazer seus exames de rotina, quanto clientes que vão acompanhar diversas patologias, com níveis de gravidade diferentes. Ao analisarmos quais condutas do enfermeiro diante de possíveis complicações, que podem ser observadas no retorno do cliente para uma recoleta, as sujeitas de pesquisas relatam que:

"Primeiramente cabe a análise
do evento adverso, assim como as
orientaçôes adequadas ao cliente.
Realizar demais encaminhamentos
(avaliação médica), quando neces-
sário. E, Posteriormente, registrar o
evento adverso e repassar as orien-
taçôes ao colaborador responsável e
a equipe." (Enfermeira 1)

"Avaliar o local com Flebite, se for algo recente, orientar quanto a compressas frias nos primeiros momentos e próximos dias compressas mornas. Avaliar a flexibilidade do membro, movimentos, se houve comprometimento de nervos, tensões, e seguir orientação acompanhamento com o cliente. Se preciso comunicar o médico responsável para avaliar. Procurar saber quem foi o coletador, conversar e repassar o caso para que fique ciente da situação." (Enfermeira 8)

Habitualmente, os hematomas que surgem após a liberação do cliente da coleta estão relacionados à falha na compressão local, quando o paciente dobra o braço 
imediatamente após a punção. A lesão pode causar desconforto e dor, mas a simples visualização do hematoma pode gerar grande ansiedade e descontentamento. $\mathrm{O}$ paciente pode associar um hematoma a uma experiência negativa de punção venosa e hesitar em fazer exames de sangue no futuro.

A análise dos eventos adversos envolve a verificação das condutas do procedimento de coleta, a enfermagem avalia os tipos de eventos adversos que possibilitam melhoria no processo e, consequentemente, da qualidade da assistência prestada ao paciente, com a possível elaboração de indicadores para a assistência de enfermagem, que impactem no aperfeiçoamento e melhoria dos processos de gestão $0^{(11)}$.

Os laboratórios de análises clínicas, por ser considerado um local que presta atendimento de saúde e possui um fluxo expressivo de clientes portando os mais variados acometimentos em saúde, acarretando muitas vezes mudança de rotina, como a necessidade de jejum prolongado, descontinuidade das medicações, e o fator emocional gerado pela preparação para o exame e a ansiedade pelos resultados, podem levar a uma exacerbação do quadro, necessitando de cuidados imediatos para estabilizar o estado de saúde do paciente, podendo ser referenciado a um cuidado adequado. Diante destas possibilidades, o profissional que presta assistência precisa ter conhecimento para tal intervenção. $\mathrm{O}$ enfermeiro atua como principal interventor nas situações de urgência e emergência, como verifica-se nas falas abaixo:

"Sim, além da equipe de coleta. Avaliar a gravidade da intercorrência e tomar as medidas cabiveis para a situação." (Enfermeira 4)

"Sim, primeiramente se identificar, se informar da situação, acalmar o cliente, aferir sinais vitais (PA, FC, FR), controlar situação de sinais e sintomas, se preciso alimentar, e caso haja necessidade pedir socorro para SAMU (bombeiros) ou qualquer outro apoio emergencial." (Enfermeira 6)

\section{Desta forma,}

é importante

considerar que a

fase pré-analítica

concentra a maior

parte dos equívocos

que podem gerar

resultados não

consistentes com o

quadro clínico do

paciente. Estima-

se que problemas

nessa etapa sejam

responsáveis por

cerca de $70 \%$ dos

erros ocorridos nos

laboratórios.
Segundo o COFEN ${ }^{(12)}$, em sua Resolução n. ${ }^{\circ} 146$, o enfermeiro está adequadamente inserido no atendimento destes agravos e possui amparo legal segundo suas normativas, em âmbito nacional, a obrigatoriedade de haver Enfermeiros em todas as unidades de serviços nos quais são desenvolvidas ações de Enfermagem que envolvam procedimentos de alta complexidade, comuns na assistência a pacientes críticos/ potencialmente críticos. Adicionalmente, incluem-se entre as responsabilidades privativas do Enfermeiro, cuidados diretos de enfermagem a pacientes graves com risco de vida e cuidados de enfermagem de maior complexidade técnica e que exijam conhecimentos científicos adequados e capacidade de tomar decisóes imediatas; e, como integrante da equipe de saúde, a participação nos programas e nas atividades de assistência integral à saúde individual e de grupos específicos, particularmente daqueles prioritários e de alto risco.

Desta forma, é importante considerar que a fase pré-analítica concentra a maior parte dos equívocos que podem gerar resultados não consistentes com o quadro clínico do paciente. Estima-se que problemas nessa etapa sejam responsáveis por cerca de $70 \%$ dos erros ocorridos nos laboratórios. Dentre eles, vale destacar os aspectos relacionados à orientação do paciente, como a necessidade ou não do jejum e o intervalo adequado deste, o tipo de alimentação, a prática de exercício físico, o uso de medicamentos capazes de interferir na análise e mudanças abruptas nos hábitos da rotina diária precedendo a coleta ${ }^{(13)}$.

Com isso, é necessário total atenção com o cliente e procedimento, para garantir a qualidade e a satisfação do mesmo. No momento da coleta tem que ter uma atenção, em todo processo, pois nele que se dá a satisfação e construção do vínculo com o cliente, em seguida o material vai ser acondicionado corretamente conforme diretrizes vigentes até o envio das amostras.

- Fase analítica: No que se refere às atribuições do enfermeiro na fase analítica, foi relatado pelas sujeitas de pesquisa que o enfermeiro 
contribui para a aplicação dos testes rápidos:

"O enfermeiro pode participar, no caso da realização de testes rápidos; procedimentos de TS's e TC's, entre outros." (Enfermeira 1)

"Sim, realizamos a leitura de $P P D$ que é um teste para triagem de $T B$, realizamos alguns testes de coagulação e glicemia capilar." (Enfermeira 5)

O teste de Tempo de Sangramento (TS), assim como o de Tempo de Tromboplastina Parcial Ativado (TTPA), Tempo de Protrombina (TP), Tempo de Trombina (TT) e Dosagem de Fibrinogênio, são exames que avaliam o perfil de coagulação de indivíduos na ocasião de rotina pré-operatória ou ainda para investigação de doenças hemorrágicas e trombóticas ${ }^{(14)}$.

Os testes de TC e TS, caso solicitados, podem ser realizados sem preparo específico e não demonstram complexidade técni$\mathrm{ca}$, no entanto, necessitam de treinamento e capacitação profissional, visto que qualquer erro no decorrer do processo, pode interferir no resultado final ${ }^{(14)}$.

O teste de glicemia capilar é utilizado nos laboratórios de análises clínicas nas situações de urgência e emergência para descartar um descontrole metabólico de hipo ou hiperglicemia que podem ser desencadeadas por jejum prolongados, ou até mesmo, pelo paciente com patologia não tratada ou descompensada. Segundo parecer do conselho federal de enfermagem, os testes glicêmicos são realizados por tiras reagentes, e podem ser lidos visualmente por meio de tabelas de cores contidas nos rótulos dos produtos ou então, com maior precisão com o auxílio de um monitor de glicose sanguínea que fornece o resultado exato da glicemia.

Conforme evidências apresentadas, fica claro que o enfermeiro atua na fase analítica e possui competência legal para executar tais atribuições. Considerando o diagnóstico dos testes rápidos, faz-se necessária uma avaliação conjunta sem a exposição do paciente perante seu resultado laboratorial, orientando com clareza o diagnóstico e respeitando sempre o código de ética da nossa profissão.

- Fase pós-analítica: quando questionadas da participação do enfermeiro na fase pós-analítica, as sujeitas de pesquisa nos relataram que:

"O enfermeiro atua na fase pós-analitica, ao entregar os resultados de exames, garantindo a fidedignidade dos resultados aos clientes." (Enfermeira 1)

"Sim, entrega de laudos de sorologia. Controle dos indicativos da unidade." (Enfermeira 4)

O papel do profissional de enfermagem é reconhecido pela capacidade e habilidade que possui para compreender o ser humano holisticamente, pela integralidade da assistência à saúde e pela capacidade de acolher $\mathrm{e}$ identificar-se com as necessidades ${ }^{(15)}$.

Entretanto, diante de um resultado de sorologia, HIV positivo, as condutas relatadas pelas sujeitas de pesquisa incluem:

"Normalmente, os resultados de sorologia são orientados pelo enfermeiro, após contato telefônico do setor responsável com o cliente. Nesse momento, o enfermeiro, em local adequado, preservando a privacidade e sigilo do cliente, repassa as orientaçôes quanto aos resultados do exame." (Enfermeira 1)

"O enfermeiro realizada a entrega de laudo de HIV positivo, dando orientaçôes e dando suporte pra tentar tranquilizar o paciente nesse momento tão difícil." (Enfermeira 3)

Segundo a Portaria Ministerial n. ${ }^{\circ}$ $151 / 2009$, é de responsabilidade dos serviços de saúde que ofertam o diagnóstico do HIV realizar o aconselhamento, informar sobre os procedimentos a serem realizados e os possíveis resultados e garantir o sigilo e confidencialidade ${ }^{(16)}$. Considerando que existem vários fluxogramas estabelecidos de acordo com o avanço científico e com a experiência mundial consolidada, os quais permitem o diagnóstico correto da infecção pelo HIV por meio da combinação dos diferentes testes disponíveis no mercado ${ }^{(17)}$.

- Gerenciamento de enfermagem: o enfermeiro tem como função nos laboratórios de análises clínicas: a coordenação da unidade, gestão administrativa da instituição, confecção das escalas de trabalhos dos profissionais, conforme descritos pelos pesquisados em seus relatos abaixo:

"Liderar, Treinar (multiplicar)
a equipe, Coordenar a unidade,
repassar direta e indiretamente
informações a gestão e Adminis-
trativo da empresa com visão geral.
Realizar procedimentos cabiveis ao
enfermeiro, auxiliar em dúvidas a
toda equipe. E de modo geral estar
presente em todas as fases e momen-
tos que acontece no decorrer do dia-
-a-dia". (Enfermeira 6)

"Apoiar a equipe nos procedimentos realizados, realizar treinamentos contínuos, Organização de escalas de dimensionamento. Controle dos indicativos da unidade. Realizar procedimentos específicos de enfermeiro, tais como: sondagem vesical e coleta Colpocitologia". (Enfermeira 4)

"Liderar e gerenciar equipe, recursos humanos, escala de trabalho." (Enfermeira 5).

A supervisão e a coordenação são parte importante do processo de trabalho do gerente, pois trata-se de um processo educativo e contínuo que consiste, fundamentalmente, em motivar e orientar os supervisionados na execução das atividades sempre com base em normas, a fim de manter elevada a qualidade dos serviços prestados. Já a coordenação tem como finalidade harmonizar todos os atos de uma instituição, 
buscando seu sucesso, equilibrando recursos físicos, materiais e humanos. Quando as enfermeiras falam sobre a importância do trabalho do gerente de enfermagem, o fazem caracterizando o processo de trabalho, consideram cada etapa importante para a assistência como um todo ${ }^{(18)}$.

Apesar de não dominarem as teorias administrativas, os gerentes de Enfermagem demonstraram conhecimento suficiente sobre administração, o que lhes permite um bom desempenho gerencial. Dentre os aspectos considerados mais importantes no trabalho gerencial, destacam-se a garantia de condições de trabalho, um dimensionamento compatível com o serviço, supervisão da equipe de Enfermagem e organização das ações assistenciais $\left.{ }^{(19}\right)$.

Quanto à realização de educação continuada, a maioria relatou que desenvolve rotineiramente, conforme observa-se nas falas abaixo:

"A educação continuada se dá desde a capacitação de um colega/colaborador para uma nova técnica de coleta, até as orientaçóes continuas do processo. A atualização de cada Profissional, bem como as reciclagens/ treinamentos refletem diretamente no desenvolvimento e progresso na equipe em geral." (Enfermeira 1)

"Sim, trabalhos com a educação continuada, com treinamentos teóricos e práticos, sobre temas que abordamos no dia a dia em laboratório clínico." (Enfermagem 5)

Quanto às atribuições citadas como educação continuada, capacitações, treinamentos e demais orientações, encontramos evidências na literatura científica como parte das atribuições do enfermeiro orientar capacitar de forma a promover o conhecimento da sua equipe e atualizar sobre novos conhecimentos e técnicas, como também da sociedade em geral com foco na prevenção e mudanças de hábitos que promovam a saúde individual e coletiva.Um estudo afirma que a educação continuada é aquela que se realiza ao longo da vida, continuamente, sendo inerente ao desenvolvimento da pessoa e relaciona-se com a ideia de construção do ser humano e promove o aumento da capacidade de discernir e agir ${ }^{(20)}$.

A realização dessa atribuição no laboratório de análises clínicas é fundamental, visto os riscos e especificidades inerentes deste ambiente de trabalho.

\section{O esforço por parte da enfermagem em trabalhar}

com a união dos

conhecimentos

científicos à prática

é extremamente

importante, quando

se cuida de seres

humanos, devendo

observar para

além dos aspectos

clínicos, mas analisar

a consequência das

intervençôes.
A teoria de enfermagem de Virginia Henderson utilizada baseia-se nos principais questionamentos que inspiraram sua obra foram: Qual é a prática da enfermagem? Que funções específicas desempenham? E quais as atividades exclusivas do enfermeiro? ${ }^{(6)}$. Estes questionamentos filosóficos vieram de encontro com os questionamentos que a pesquisa pretendeu responder.

\section{CONSIDERAÇÕES FINAIS}

A presente pesquisa possibilitou a compreensão acerca dos cuidados de enfermagem fazendo a reflexão do que ocorre na prática com as recomendações dos órgãos competentes e pesquisas, respondendo, assim, todos os objetivos propostos deste trabalho.

O esforço por parte da enfermagem em trabalhar com a união dos conhecimentos científicos à prática é extremamente importante, quando se cuida de seres humanos, devendo observar para além dos aspectos clínicos, mas analisar a consequência das intervenções. A identificação de possíveis agravos emocionais, lesões geradas pelos procedimentos invasivos, conhecimento ineficaz sobre o procedimento e seu quadro de saúde, que podem levar a resultados imprecisos, acarretando ao cliente um tratamento diferente das necessidades e, consequentemente, a uma resposta ao tratamento indesejada.

Analisando os resultados do trabalho demonstrou-se a importância do profissional enfermeiro, frente ao julgamento e execução dos cuidados prestados no atendimento assistencial do laboratório de análises clinicas. As pesquisadas descreveram a sua participação nos processos que compõem a rotina dos laboratórios, dentro das quais foi observado que, faz parte algumas atribuições privativas do enfermeiro, como gasometria, sondagem vesical de alívio, colpocitologia, gerenciamento da equipe de enfermagem, cuidados diretos ao paciente nas intercorrências, treinamento da equipe de enfermagem e outras que, no âmbito privado da institui- 


\section{artigo}

Prigoli, J.; Costa, V.; Souza, G.F.S.; Branco, C.C.S.S.P.; Rodrigues, M.M.; Rosa, T.;

Atuação do enfermeiro em laboratório de análises clínicas

ção, eram medicações relacionadas a hormônios, sobre as quais demonstraram conhecimento e estão vinculadas à formação de enfermagem e suas atribuições.

Mesmo esse setor sendo multiprofissional, evidenciou-se a importância do profissional enfermeiro, e que este, participa das três fases distintas do processo, na fase pré-analítica atuam gerenciado a equipe de enfermagem, na logística do laboratório, materiais e insumos, na coleta de materiais quando a atividade é privativa do enfermeiro e no armazenamento. Já na fase analítica descreveram que realizavam alguns procedimentos, como testes rápidos. $\mathrm{Na}$ fase pós-analítica realizam a emissão de laudos, entrega de testes de HIV incluindo o acon- selhamento ao cliente, sendo assim, participam de todas as fases descritas no processo.

As capacitaçôes e atualizações que os profissionais recebem são fornecidas pela instituição em cursos ministrados por enfermeiros, onde é designado um profissional para essa função exclusiva. Quanto à base teórica, ela é escassa, acarretando a busca em protocolos internacionais. A falta de base teórica para a enfermagem e de portarias específicas para a atuação do enfermeiro em laboratório de análises clínicas foram obstáculos que encontramos para tornar a pesquisa mais completa; da mesma forma, o curto tempo que os pesquisadores tiveram para desenvolver a análise foi a principal dificuldade en- contrada. Por fim, evidenciou-se que esta pesquisa é importante e contribui para o conhecimento sobre as atribuições do enfermeiro neste setor, profissionais, acadêmicos de ensino superior e profissionais atuantes na área da saúde que busquem por estudar o tema e fortalecer o conhecimento na área, que vem crescendo e abrindo portas para a enfermagem.

Foi observado que as pesquisadoras não mencionaram nas suas respostas sobre a prática da Sistematização da Assistência de Enfermagem (SAE) nos laboratórios. Por ser preconizada pelo COFEN a sua implementação, fica uma lacuna sobre o tema e sua prática nestas instituições, podendo futuramente ser tema de pesquisa.

\section{REFERÊNCIAS}

1. Guimarães $\mathrm{H}$, et al. O laboratório clínico e os erros pré-analíticos. Revista HCPA. 2011;31(1):66-72.

2. Silva MO. Plano educativo. In: Diabetes mellitus: clínica, diagnóstico, tratamento multidisciplinar. São Paulo: Editora Atheneu; 2004.

3. Agência Nacional de Vigilância Sanitária (BR). Resolução RDC n. ${ }^{\circ} 302$ - Regulamento técnico para funcionamento de laboratórios clínicos. Brasília (DF): ANVISA; 2005.

4. Brasil. Decreto n. ${ }^{\circ} 94.406$, de 08 de junho de 1987. Regulamenta a Lei . $^{\circ} 7.498$, de 25 de junho de 1986, que dispõe sobre o exercício profissional da enfermagem, e dá outras providências. Brasília (DF): Gabinete do Ministro; 1987.

5. Bardin L. Análise de conteúdo. São Paulo: Edições 70; 2011.

6. Armijo PP. Henderson's theory and its application in advanced nursing care in a pediatric ward. Medwave. 2012 nov.; 12(10).

7. Silva AM, Peduzzi M. O trabalho de enfermagem em laboratórios de análises clínicas. Rev. Latino-Am. Enfermagem. 2005;13(1):65-71.

8. Agência Nacional de Vigilância Sanitária (BR). Resolução RDC n. ${ }^{\circ}$ 306. Dispõe sobre o Regulamento Técnico para o gerenciamento de resíduos de serviços de saúde. Agência Nacional de Vigilância Sanitária. Brasília (DF): ANVISA; 2004.

9. Costa ARN, Leal D, Kerber RF. Riscos ocupacionais em trabalhadores de laboratórios de análises clínicas. [Monografia] Pós-Graduação em Medicina do Trabalho. Universidade Federal de Santa Catarina (SC); 2001.

10. Riques DC, Caíres FMA. Criança Hospitalizada 2014: Manual de Orientação aos pais. Sociedade Brasileira de Psicologia; 2014.

11. Almeida $\mathrm{ML}$, et al. Instrumentos gerenciais utilizados na tomada de decisão do enfermeiro no contexto hospitalar. Texto contexto enferm, 2011;20(Spe):131-137.
12. Conselho Federal de Enfermagem. Resolução n. ${ }^{\circ}$ 146, de 12 junho de 1992. Dispõe sobre a obrigatoriedade de haver profissional de enfermagem em todos os locais que exerçam atividade de enfermagem. Rio de Janeiro (RJ): COFEN; 1992. p. 112-4.

13. Goswami B, et al. Evaluation of errors in a clinical laboratory: a one- year experience. Clin Chem Lab Med. 2010;48(1):63-6.

14. Sociedade Brasileira de Patologia Clínica e Medicina Laboratorial. Coleta e Preparo da Amostra Biológica. Barueri: Manole; 2014.

15. Costa VG, Moreli ML. Principais parâmetros biológicos avaliados em erros na fase pré-analítica de laboratórios clínicos: revisão sistemática. J Bras Patol Med Lab. 2012 jun.;48(3):163-168.

16. Ministério da Saúde (BR). Portaria n. ${ }^{\circ}$ 151, de 14 de outubro de 2009. Considerando a necessidade de se criar alternativas para a ampliação do acesso ao diagnóstico da infecção pelo HIV. Em atendimento aos princípios da equidade e da integralidade da assistência, bem como da universalidade de acesso aos serviços de saúde do Sistema Único de Saúde - SUS. Brasília (DF): MS; 2009.

17. Ministério da Saúde (BR). Portaria n. ${ }^{\circ} 29$, de 17 de dezembro de 2013. Aprova o Manual Técnico para o Diagnóstico da Infecção pelo HIV em Adultos e Crianças e dá outras providências. Brasília (DF): MS; 2013.

18. Damasceno C. O trabalho Gerencial da Enfermagem: Conhecimento de

Profissionais Enfermeiros sobre suas competências gerenciais. J Nurs UFPE online. 2016 abr;10(4):1216-1221.

19. Aguiar C, Aleixo G, Barbosa JP. A Importância do Enfermeiro do na Fase Pré-Analítica de Exames Laboratoriais. Revista Científica Eletrônica de Enfermagem - FAEF. 2018 jun.;1(1).

20. Haddad S. A educação continuada e as políticas públicas no Brasil. Revista de Educação de Jovens e Adultos. 2007 ago.;1:2738. 